Letter

\title{
Correlation between Ionospheric TEC and the DCB Stability of GNSS Receivers from 2014 to 2016
}

\author{
Byung-Kyu Choi ${ }^{1, * \mathbb{C}}$, Dong-Hyo Sohn ${ }^{1}$ and Sang Jeong Lee ${ }^{2}$ \\ 1 Space Science Division, Korea Astronomy and Space Science Institute, Daejeon 34055, Korea; \\ dhsohn@kasi.re.kr \\ 2 Department of Electronics Engineering, Chungnam National University, Daejeon 34134, Korea; \\ eesj1@cnu.ac.kr \\ * Correspondence: bkchoi@kasi.re.kr; Tel.: +82-42-865-3237
}

Received: 15 October 2019; Accepted: 12 November 2019; Published: 13 November 2019

\begin{abstract}
The Global Navigation Satellite System (GNSS) differential code biases (DCBs) are a major obstacle in estimating the ionospheric total electron content (TEC). The DCBs of the GNSS receiver (rDCBs) are affected by various factors such as data quality, estimation method, receiver type, hardware temperature, and antenna characteristics. This study investigates the relationship between TEC and rDCB, and TEC and rDCB stability during a three-year period from 2014 to 2016. Linear correlations between pairs of variables, measured with Pearson's coefficient $(R)$, are considered. It is shown that the correlation between TEC and rDCB is the smallest in low-latitude regions. The mid-latitude regions exhibit the maximum value of $R$. In contrast, the correlation between TEC and $\mathrm{rDCB}$ root mean square (RMS, stability) was greater in low-latitude regions. A strong positive correlation $(R \geq 0.90)$ on average between TEC and rDCB RMS was also revealed at two additional GNSS stations in low-latitude regions, where the correlation shows clear latitudinal dependency. We found that the correlation between TEC and $\mathrm{rDCB}$ stability is still very strong even after replacing a GNSS receiver.
\end{abstract}

Keywords: GNSS; rDCB; TEC; correlation; stability

\section{Introduction}

The Global Navigation Satellite System (GNSS) receivers, which are widely distributed on the Earth's surface, are very useful for monitoring changes in the ionosphere. The ionosphere is the largest source of error in signal propagation from GNSS satellites to single-frequency GNSS receivers. Accurately estimating the total electron content (TEC) in the ionosphere can improve the position accuracy for the GNSS users. GNSS TEC can be obtained easily from the geometry-free linear combination (P1-P2 or C1-P2) using data of different frequencies. However, GNSS satellites and ground receivers have their own hardware biases, which act as large sources of error in calculating the ionospheric TEC. These hardware-associated biases still remain in the ionospheric TEC after subtracting the measurements at two different frequencies. These differences in hardware biases inherent in different GNSS code measurements are called differential code biases (DCBs). Methods for estimating the DCB have been reported in many studies [1-9].

The GNSS satellite and receiver DCB (rDCB) values are different for each satellite and receiver. In general, the DCBs of the GNSS receivers are larger than those of the GNSS satellites. GNSS satellites have DCB values of several nanoseconds (ns), while those of GNSS receivers can reach up to a few tens of ns. Wilson and Mannucci [10] demonstrated that global positioning system (GPS) satellite DCBs have long-term stability with a root mean square (RMS) error of approximately $0.2 \mathrm{~ns}$. Hernandez-Pajares et al. [11] showed that GPS satellite DCBs will remain in the range of several ns for about 10 years. 
They also observed some periodic behaviors in them, and suggested that these could be related to the ionospheric TEC.

GNSS DCBs are affected by various factors, such as data quality, estimation method, receiver type, and antenna characteristics. The diurnal variations of the $\mathrm{rDCB}$ can differ depending on the temperature of the hardware inside the receiver [12-15]. Mannucci et al. [16] suggested that the rDCB value can be more stable and more accurate when the ionospheric activity is in a somewhat quiet condition than when it is in a disturbed condition. Furthermore, Zhang et al. [17] reported that geomagnetic conditions can affect the accuracy of $\mathrm{rDCB}$ values. They noted that there was a difference in rDCB between disturbed and quiet geomagnetic conditions. Recently, Choi and Lee [18] also reported that the $\mathrm{rDCB}$ can change significantly depending on the grounding status of the antennas at GNSS sites.

The International GNSS Service (IGS) has been providing reliable Global Ionosphere Map (GIM) products since $1998[19,20]$. There are several international associate analysis centers (IAACs) such as the Center for Orbit Determination in Europe, the Jet Propulsion Laboratory, the European Space Operations Center of the European Space Agency, the Technical University of Catalonia, and the GNSS Research Center of Wuhan University. Typically GIM products are provided on a daily basis by each IAAC and IGS. In addition, they contain DCBs for the GNSS satellites and the IGS stations. DCBs and their associated RMS values are available in the daily GIM products.

The variability of DCBs related to ionospheric TEC changes has been reported by previous studies. For example, Zhang et al. [21] reported that DCBs are related to ionospheric variability. Considering the basic ionospheric approximation in the DCB estimation approach, they suggested that the day-to-day variation of GPS satellite DCBs is associated with the strength of the ionospheric day-to-day variation. Themens et al. [22] investigated that the behavior of GPS rDCB in the high-latitude region shows seasonal and temperature variability. In addition, ionospheric gradients at middle- and low-latitude regions can generate seasonal and solar-cycle variations of $\operatorname{rDCB}[23,24]$. However, a few case studies of DCB variability related to ionospheric change have been conducted in low-latitude or high-latitude regions.

In this study, we focus on the relationship between the stability of rDCB and ionospheric TEC change with solar activity during a period of three years from 2014 to 2016. This period corresponds to solar cycle 24 , and has a gradual decrease in the number of sunspots. The stability of the rDCB is analyzed in terms of rDCB RMS value, which can be obtained from the European Space Agency hourly rapid GIM (EHRG) products. Using the EHRG GIMs, we investigate vertical total electron content (VTEC), rDCB, and rDCB RMS values for the GNSS stations in three different latitude regions. In addition, correlation coefficients are considered to measure the linear relationships between pairs of variables (VTEC and rDCB, VTEC and rDCB RMS).

\section{Data and Methodology}

EHRG GIM products are generated on a daily basis using data from about 220 GNSS stations of the IGS. They are modeled using the ionospheric model, based on spherical harmonic expansion up to degree and order 15, which are provided by the IGS. GIM products can be downloaded via the Crustal Dynamic Data Information System server (ftp://cddis.gsfc.nasa.gov/). As of 2014, EHRG GIM products provided in IONosphere EXchange (IONEX) format have the vertical TEC (VTEC) with a temporal resolution of 1 hour, and a spatial resolution with $2.5^{\circ}$ in latitude and $5^{\circ}$ in longitude, respectively. They also include the P1-P2 DCB for all GPS satellites, and rDCB for ground stations for each day.

Ionospheric TEC can be calculated from the geometry-free linear combination using dual-frequency GNSS measurements. The electron density in the ionosphere along the line of sight from a satellite to receiver is referred to as TEC. It can be directly calculated using Equation (1).

$$
\text { STEC }=\frac{1}{40.3}\left(\frac{f_{1}^{2} \cdot f_{2}^{2}}{f_{1}^{2}-f_{2}^{2}}\right)\left(P_{2}-P_{1}+d^{s}+d_{r}\right)
$$


where STEC represents the slant TEC. $P_{1}$ and $P_{2}$ are GNSS pseudoranges. $f_{1}$ and $f_{2}$ are the frequencies of GNSS signals. $d^{s}$ and $d_{r}$ are GNSS satellite DCB and receiver DCB, respectively. To estimate the GNSS satellite and receiver DCBs, constraint condition is required to separate the satellite and receiver DCBs. It is assumed that the sum of all available GNSS satellite DCBs is zero.

In general, GIMs use the spherical harmonic expansion method to derive the spatial distribution of ionospheric TEC. The spherical harmonic expansion technique is shown in Equation $(2)[25,26]$.

$$
\operatorname{VTEC}(\beta, s)=\sum_{n=0}^{N} \sum_{m=0}^{n} \bar{P}_{n m}(\sin \beta)\left(\bar{C}_{n m} \cos (m s)+\bar{S}_{n m}=1 / F \cdot\left[S T E C-d^{s}-d_{r}\right]\right.
$$

where, VTEC is the vertical TEC, $\beta$ and $s$ are the geocentric latitude and solar-fixed longitude of ionospheric pierce points, respectively. $N$ denotes the maximum degree of the spherical harmonic expansion. $\bar{P}_{n m}$ represents the normalized associated Legendre function with the degree $n$ and the order $m . \bar{C}_{n m}$ and $\bar{S}_{n m}$ are the TEC unknown coefficients of spherical harmonics. $F$ is a slant factor. STEC is converted to VTEC by a slant factor.

The sunspot number associated with solar activity was analyzed to investigate the relationship between the stability of rDCB and changes in the ionospheric TEC. Figure 1 shows the daily sunspot numbers during the period from 2011 to 2016. Data on sunspot numbers is available at http: //www.sidc.be/silso/ssngraphics. In Figure 1, the yellow solid line indicates the daily sunspot number and the red solid line represents the smoothed daily sunspot number with sinusoidal harmonic curve fitting. Within solar cycle 24, solar activity reached its maximum in April 2014 and has weakened in the number of sunspots since then. In the present study, we consider ionospheric TEC changes and rDCB stability in a period of weakening solar activity from 2014 to 2016.

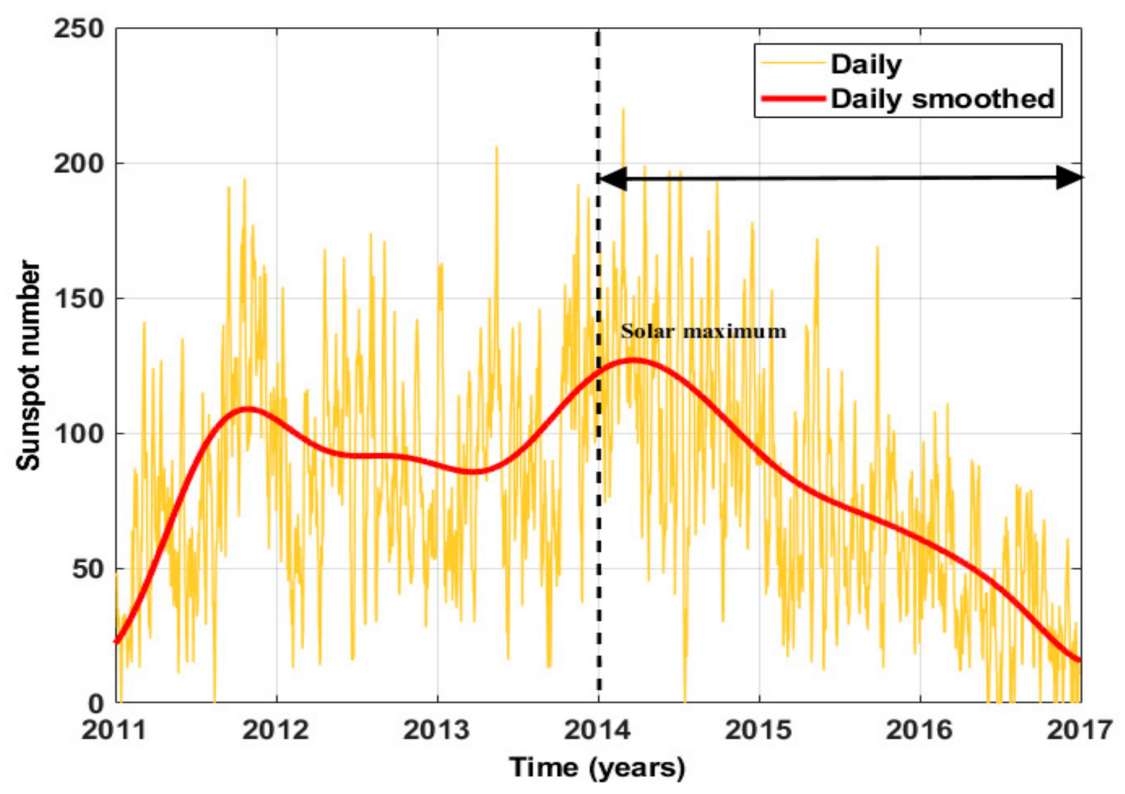

Figure 1. The number of sunspots from 2011 to 2016. The yellow solid line shows daily sunspot numbers. The red solid line indicates the smoothed daily sunspot numbers. The vertical black dotted line represents the beginning of 2014. Solar cycle 24 reached its maximum in April 2014.

Figure 2 shows the locations of the six GNSS reference stations selected to investigate the relationship between ionospheric TEC and rDCB, and between ionospheric TEC and rDCB stability. The locations of GNSS reference stations are considered in terms of three latitude zones: low latitude $\left(20^{\circ} \mathrm{S}-20^{\circ} \mathrm{N}\right)$, mid-latitude $\left(20^{\circ} \mathrm{S}-60^{\circ} \mathrm{S}, 20^{\circ} \mathrm{N}-60^{\circ} \mathrm{N}\right)$ and high latitude $\left(60^{\circ} \mathrm{S}-90^{\circ} \mathrm{S}, 60^{\circ} \mathrm{N}-90^{\circ} \mathrm{N}\right)$, respectively. There are two GNSS stations in each latitude zone. Table 1 shows the receiver and antenna types at the six GNSS reference stations from 2014 to 2016. The IGS network consists of more than 500 
GNSS stations distributed around the world. All selected stations in this study are included in the IGS network. We used two major considerations when selecting these GNSS stations. First, the GNSS receivers and antennas should not be replaced during the three years from 2014 to 2016. Second, the DCBs should be reliably provided with daily values in GIM products. In addition, different GNSS receiver models were considered to exclude receiver-type dependency. Other GNSS sites, except for NKLG and WILL, are equipped with different receivers as listed in Table 1.

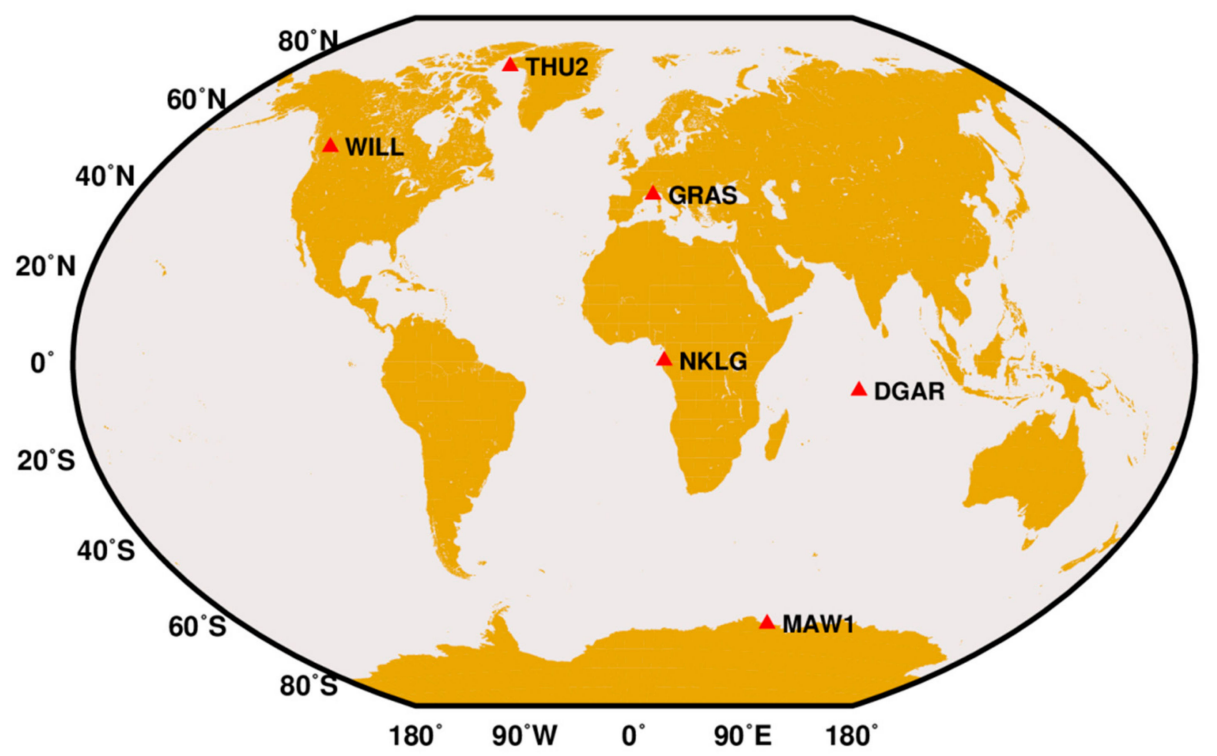

Figure 2. Geographic locations of the six Global Navigation Satellite System (GNSS) reference stations, which are marked by red triangles.

Table 1. The receiver and antenna types at the six selected GNSS stations from 2014 to 2016.

\begin{tabular}{cccccc}
\hline Site Name & Receiver Type & \multicolumn{2}{c}{ Antenna Type } & $\begin{array}{c}\text { Geographic } \\
\text { Latitude (Degrees) }\end{array}$ & $\begin{array}{c}\text { Geographic } \\
\text { Longitude (Degrees) }\end{array}$ \\
\hline DGAR & ASHTECH UZ-12 & ASH701945E_M & NONE & $7.2 \mathrm{~S}$ & $72.3 \mathrm{E}$ \\
NKLG & TRIMBLE NETR9 & TRM59800.00 & SCIS & $0.3 \mathrm{~N}$ & $9.6 \mathrm{E}$ \\
GRAS & TRIMBLE NETR5 & ASH701945E_M & NONE & $43.7 \mathrm{~N}$ & $6.9 \mathrm{E}$ \\
WILL & TRIMBLE NETR9 & TRM59800.00 & SCIS & $52.2 \mathrm{~N}$ & $122.1 \mathrm{~S}$ \\
THU2 & JAVAD TRE_G3T SIGMA & TPSCR3_GGD & NONE & $76.5 \mathrm{~N}$ & $68.8 \mathrm{~S}$ \\
MAW1 & LEICA GRX1200GGPRO & ASH701073.1 & SCIS & $67.6 \mathrm{~S}$ & $62.8 \mathrm{E}$ \\
\hline
\end{tabular}

\section{Results}

Figure 3 shows the time series of vertical TEC units (VTEC) (top panel), rDCB (middle panel), and rDCB RMS (bottom panel) for each GNSS station from 2014 to 2016. The amplitude of the VTEC values at all stations (DGAR, NKLG, GRAS, WILL, MAW1, and THU2) decreased gradually with solar activity. The rDCB RMS values are multiplied by the number 50 because their values are too small. Therefore, we can see clear variations in the rDCB RMS 50 time series. Figure $3 a, b$ show the time-dependent changes of the above quantitative variables at the DGAR and NKLG stations, which are located at the Earth's equatorial region. The data curves at mid-latitudes (GRAS and WILL) and higher latitudes (MAW1 and THU2) are shown in Figure 3c-f. The most dominant feature in the VTEC, rDCB, and rDCB RMS*50 time series is the annual frequency component. A strong semiannual component is also observed in low- and mid-latitude regions. Zhao et al. [27] investigated TEC climatology from the global ionosphere maps; they reported that the semiannual behavior of the ionospheric TEC is more significant in low-latitude regions than in high-latitude regions, which is consistent with our results. As a matter of fact, the amplitude of VTEC at MAW1 in the Southern Hemisphere is higher 
than that at THU2 in the Northern Hemisphere. Therefore, at high-latitudes, the rDCB does not seem to be significantly correlated with TEC variations.

A polynomial fitting method with 10 degrees is considered to show clear periodic features in the data time series. As shown in Figure 3, the magnitude of the VTEC is normally larger in low-latitude regions, while it is smaller in higher latitude regions. The effect of solar activity on ionospheric TEC can be stronger at low latitudes than at mid- or high-latitudes. At all stations, periodic variations in the rDCB and rDCB RMS*50 time series are similar to changes in the VTEC time series.

To investigate the relationship between the two different variables, a Pearson correlation was considered. The Pearson correlation is the most widely used to find the linear relationship between normally distributed variables. Therefore, Pearson's coefficient was used to calculate the linear relationship between two variables according to the following Equation (3).

$$
\rho(A, B)=\frac{1}{N-1} \sum_{i=1}^{N}\left(\frac{\overline{A_{i}-\mu A}}{\sigma A}\right)\left(\frac{\overline{B_{i}-\mu B}}{\sigma B}\right)
$$

where $\mu A$ and $\sigma A$ are the mean and standard deviation of the variable $A$, respectively. $\mu B$ and $\sigma B$ are the mean and standard deviation of the variable $B$. The index, $R$, which indicates the correlation between the two variables, is calculated by the following Equation (4).

$$
R=\left(\begin{array}{cc}
\rho(A, A) & \rho(A, B) \\
\rho(B, A) & \rho(B, B)
\end{array}\right)
$$

Since the variables $A$ and $B$ always have a direct correlation to themselves, the diagonal elements $\rho(A, A)$ and $\rho(B, B)$ of the matrix are necessarily equal to one. $R$ is simply defined as $\rho(A, B)$. Several approaches have been suggested to interpret the correlation coefficient with respect to different cutoff points. In this study, we choose a classification proposed by Schober et al. [28]. They categorised the absolute magnitude of the correlation coefficient into five categories as follows: $0.00-0.10$ (negligible correlation), $0.10-0.39$ (weak correlation), $0.40-0.69$ (moderate correlation), $0.70-0.89$ (strong correlation), and $0.90-1.00$ (very strong correlation).

Figure 4 shows different correlation coefficients between $\mathrm{rDCB}$ and VTEC, and between $\mathrm{rDCB}$ RMS and VTEC, respectively. In Figure 4a-f, the upper panels show the correlation between rDCB and VTEC, while the lower panels show the correlation between rDCB RMS and VTEC. The value of $R$ between $\mathrm{rDCB}$ and VTEC ranges from 0.33 to 0.78 , while $R$ between rDCB RMS and VTEC varies from 0.50 to 0.94 .

Two GNSS stations (DGAR and NKLG) in the equatorial regions showed a positive linear relationship between rDCB and VTEC. Their $R$ values were 0.33 and 0.40 , respectively. According to the classification defined by Schober et al. [28], the DGAR and NKLG stations can be interpreted as having a weak or moderate positive correlation between the two variables. On the other hand, rDCB RMS*50 and VTEC are highly correlated. As shown in Figure $4 \mathrm{a}, \mathrm{b}$, the $R$ values for this correlation are 0.87 and 0.94 , respectively. In mid-latitude regions (GRAS and WILL), the $R$ values between VTEC and $\mathrm{rDCB}$ are 0.78 and 0.73 , respectively. This indicates that there is a strong correlation between the two variables in mid-latitude regions. In addition, the relationship between VTEC and rDCB RMS 50 at mid-latitudes was investigated. The $R$ values for this correlation were 0.60 and 0.68 , respectively. We can see that there is a clear difference between these variables in the low-latitude and mid-latitude regions. The correlation between ionospheric TEC and $\mathrm{rDCB}$ stability in low-latitude regions is higher than that in mid-latitude regions. Conversely, the correlation between ionospheric TEC and rDCB is relatively higher in mid-latitude regions. 


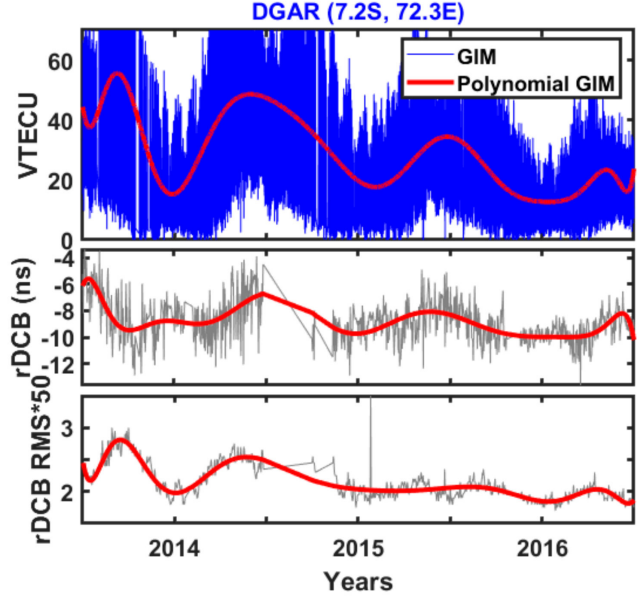

(a)
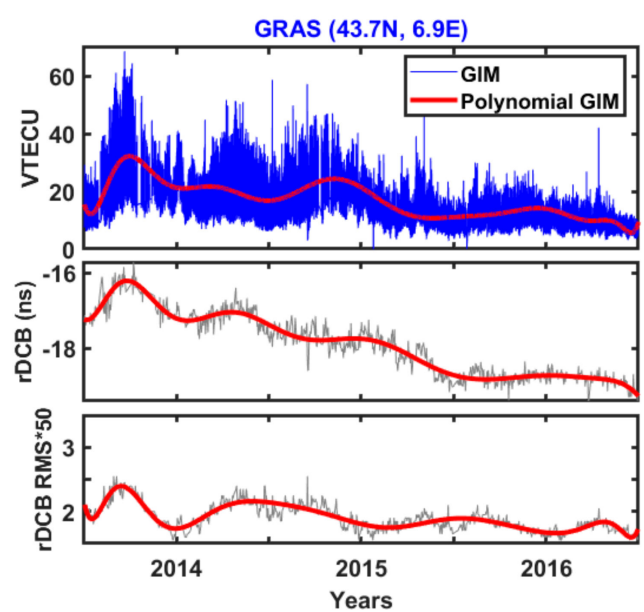

(c)
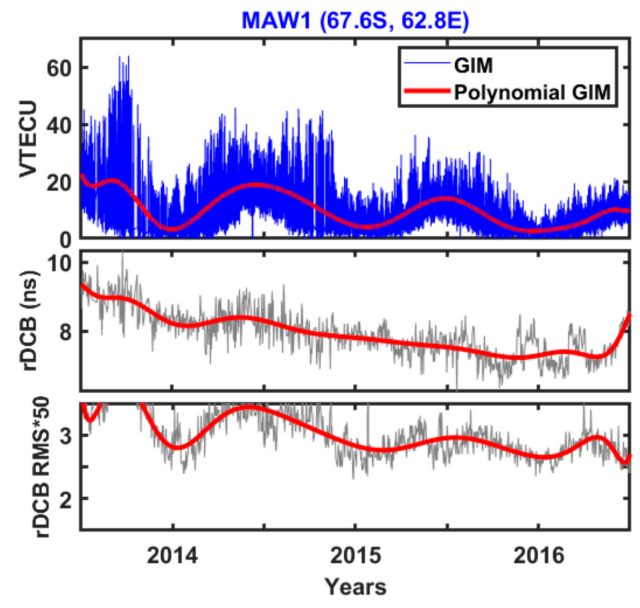

(e)
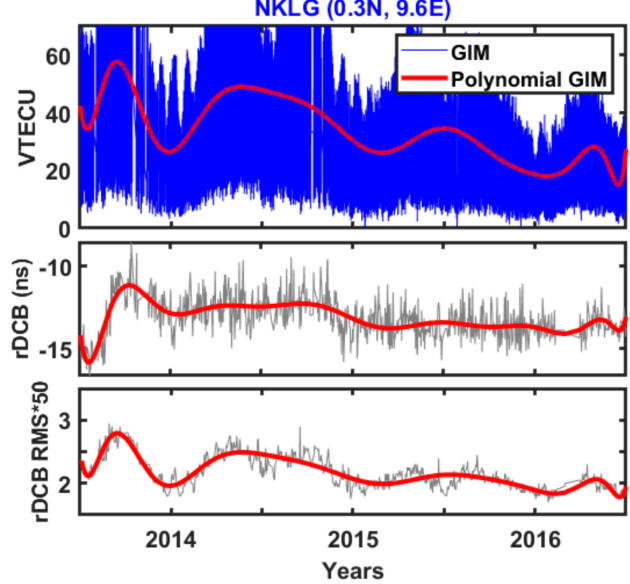

(b)

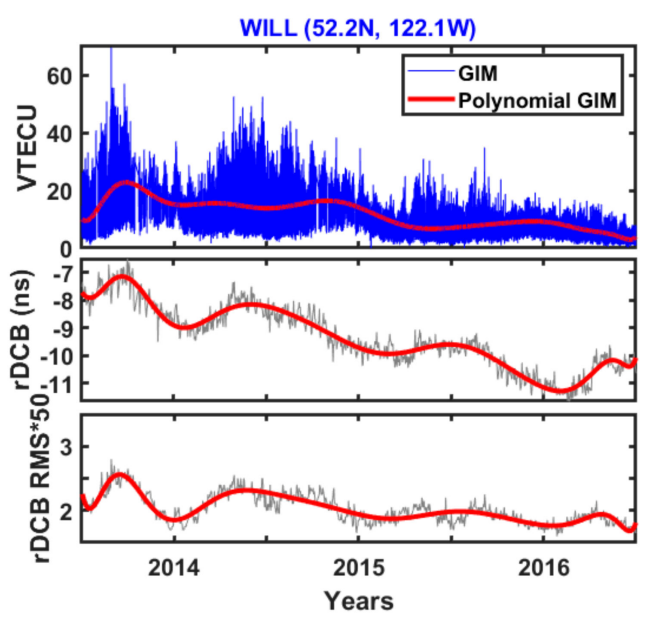

(d)

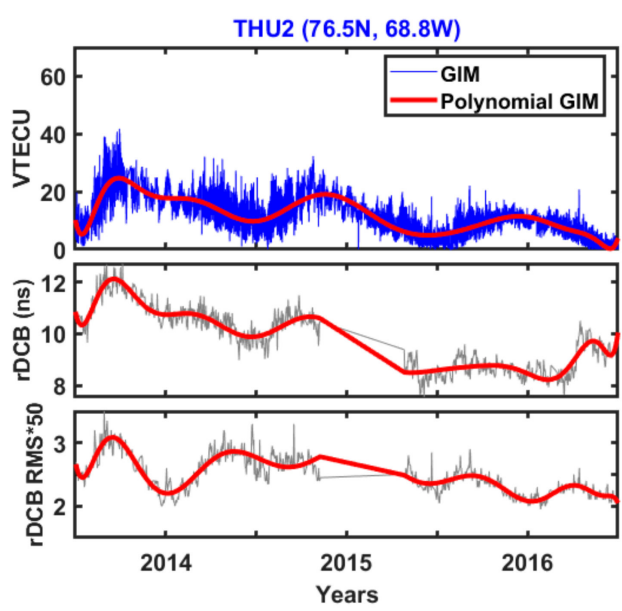

(f)

Figure 3. Time series of vertical total electron content (VTEC), receiver differential code bias (rDCB), and rDCB root mean square (RMS)*50 from 2014 to 2016 at each GNSS reference station: (a) DGAR, (b) NKLG, (c) GRAS, (d) WILL, (e) MAW1, and (f) THU2. In all panels, the red solid lines represent the polynomial fitting of the data. The gray lines denote the raw data. 
To investigate the latitudinal dependency of the correlation in more detail, we considered two additional GNSS stations (MAW1 and THU2) in high-latitude regions. As shown in Figure 4e,f, the two stations show different correlations. At MAW1 station in the southern hemisphere, the correlation between VTEC and rDCB was slightly smaller than that between VTEC and rDCB RMS. In contrast, the THU2 site in the highest latitude of the northern hemisphere, the correlation $(R \sim 0.64)$ between VTEC and rDCB was a little larger than that $(R \sim 0.50)$ between VTEC and rDCB RMS. As a result, correlations between VTEC and rDCB RMS are closely associated with a latitudinal dependency. $R$ between VTEC and rDCB is the smallest at low-latitude regions. The mid-latitude regions exhibit the maximum value of $R$. Ionospheric TEC variability in mid-latitude is generally less irregular compared to that in low and high latitudes. The estimation of rDCB at mid-latitude is also expected to be stable. It can mainly be associated with the effect of ionospheric irregularities like rapid plasma fluctuation, which is known as ionospheric scintillation. Ionospheric irregularities occur more often in low- and high-latitudes than in mid-latitudes [29]. They can affect the amplitude and phase of GNSS signals rapidly and seem to degrade the accuracy of rDCB. Therefore, the correlation between VTEC and rDCB itself at mid-latitude may be relatively higher than that at low and high latitudes. On the other hand, $R$ between VTEC and rDCB RMS*50 becomes smaller on average in high-latitude regions. The peak value of $R$ is found in low-latitude regions. The latitude-dependent tendency for these correlations is clearly seen in the bottom panels of Figure 4a-f.

To further analyze the latitudinal dependency of the correlation between VTEC and rDCB RMS in more detail, data from the 7 additional GNSS stations were examined. The receiver and antenna types at the 7 additional GNSS stations from 2014 to 2016 are listed in Table 2. Figure 5 shows the locations of the 13 GNSS stations and the $R$ values between VTEC and rDCB RMS for three years. The numbers in parentheses in Figure 5 represent the $R$ values. We can also see the apparent latitudinal dependency from the $R$ values of the 13 GNSS stations. The three GNSS stations (NKLG, DGAR, and DARW) at low latitudes have large $R$ values above 0.86 . In particular, as the latitude increases from the equator, the $R$ value decreases slightly. There are 6 GNSS stations (WILL, GRAS, JPLM, ULAB, SUWN, and CORD) in mid-latitude regions. Their $R$ values range from 0.6 to 0.8 . The averaged value of $R$ is approximately 0.69 , which is smaller than that on average in low-latitude regions. The four GNSS stations (THU2, SCOR, MAW1, and CAS1) at high latitudes have weak correlations compared to those at low and mid-latitudes. The $R$ value ranges from 0.49 to 0.61 . Interestingly, the $R$ values in high-latitudes of the Southern Hemisphere were greater than those in high-latitudes of the Northern Hemisphere. $R$ between VTEC and rDCB RMS can be associated with the magnitude of TEC following the latitudinal dependency. As shown in Figure $3 \mathrm{e}-\mathrm{f}$, the magnitude of TEC is higher at the Southern Hemisphere compared to the Northern Hemisphere during three years from 2014 to 2016. Therefore, the asymmetry of the ionosphere in different hemispheres can affect $R$ between VTEC and rDCB RMS.

The ionosphere over equatorial and low-latitude regions can be strongly influenced by phenomena such as daytime spread- $F$, equatorial ionization anomalies, and intense irregularities in TEC distributions. These effects in the equatorial and low-latitude regions can affect GPS signals passing through the ionosphere. In particular, the dynamically changing ionosphere in the equatorial regions may have a large effect on the stability of the receiver DCB. Therefore, a strong ionospheric activity might induce a high correlation between TEC and receiver DCB stability.In high-latitude regions, however, the intense ionospheric TEC irregularities are presumed to be caused by auroral activities and high-speed plasma convection [30]. The magnitude of TEC at high latitudes is about three times smaller than that at low latitudes [31]. The correlation between TEC and rDCB in low-latitude regions is smaller than that in high-latitude regions. On the other hand, the amplitude of TEC decreases with higher latitudes. This may also be associated with rapid TEC changes, or to the magnitude of TEC. 


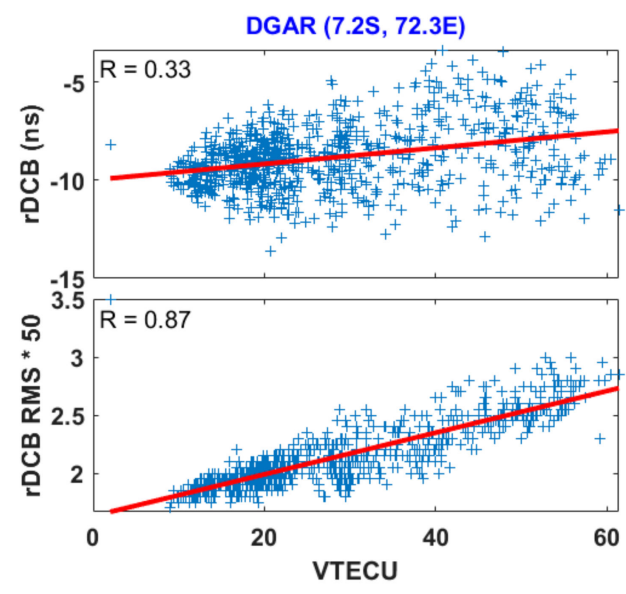

(a)

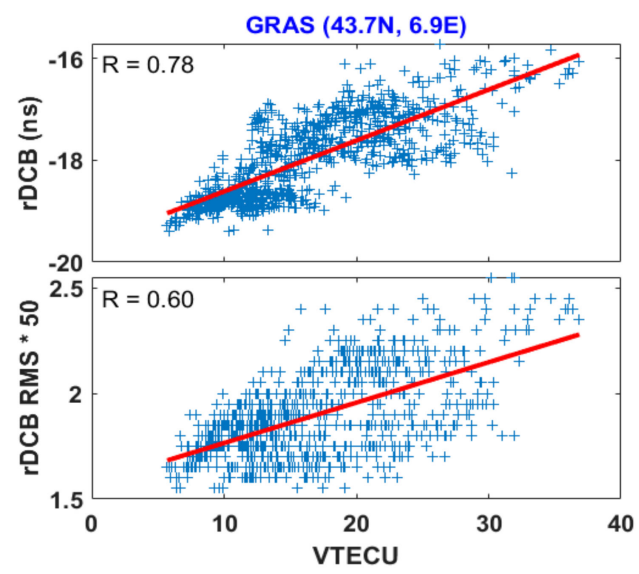

(c)

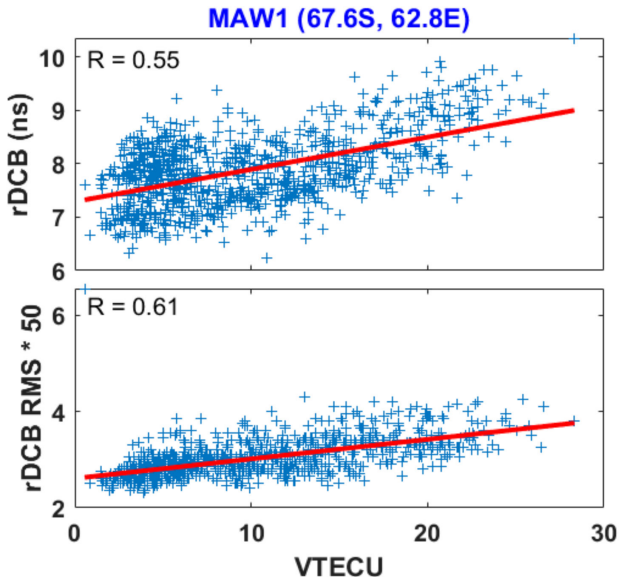

(e)

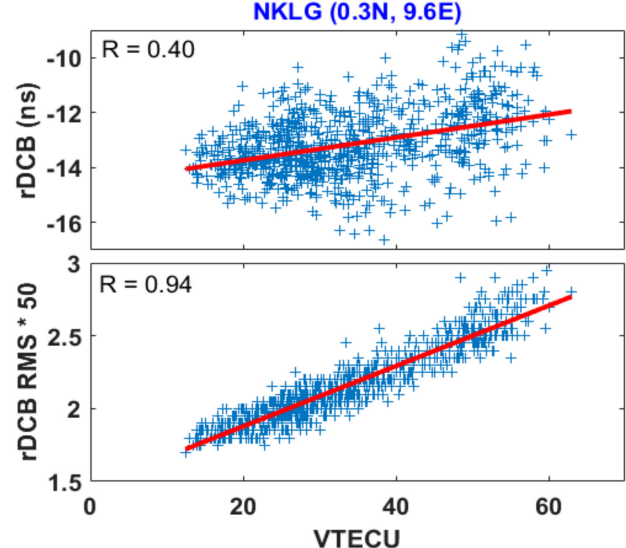

(b)

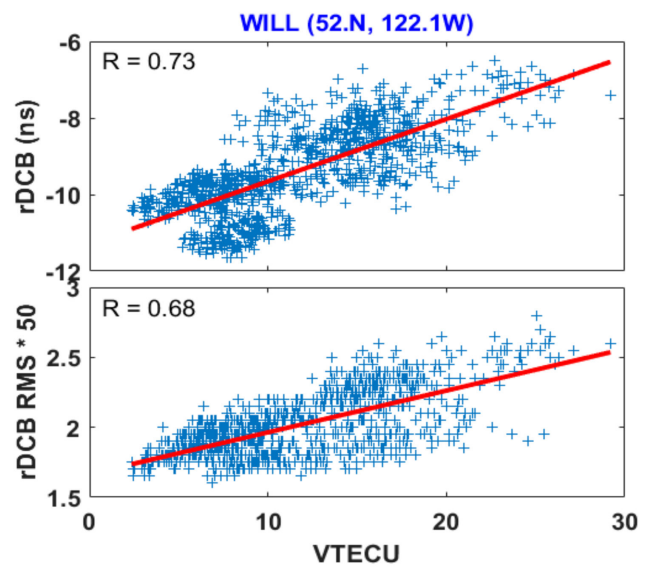

(d)

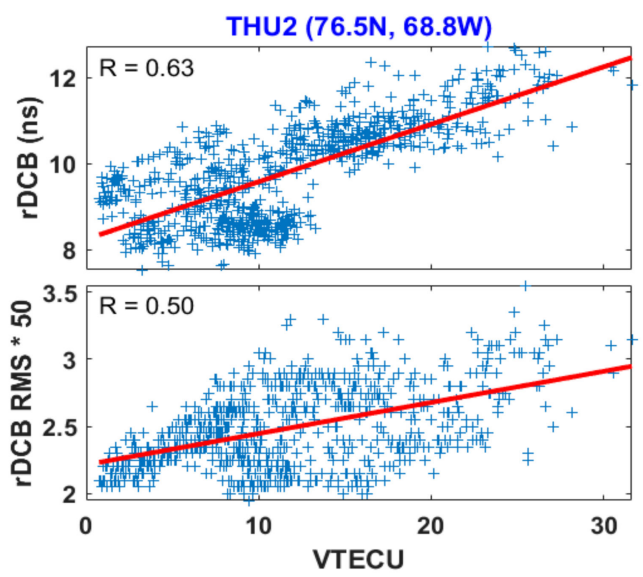

(f)

Figure 4. Pearson's orrelation coefficients $(R)$ between two variables (rDCB and VTEC, rDCB RMS and VTEC) calculated at the 6 GNSS stations using data from 2014 to 2016: (a) DGAR, (b) NKLG, (c) GRAS, (d) WILL, (e) MAW1, and (f) THU2. The red solid lines are fitted by a linear regression model that minimizes the sum of the squared residuals. 


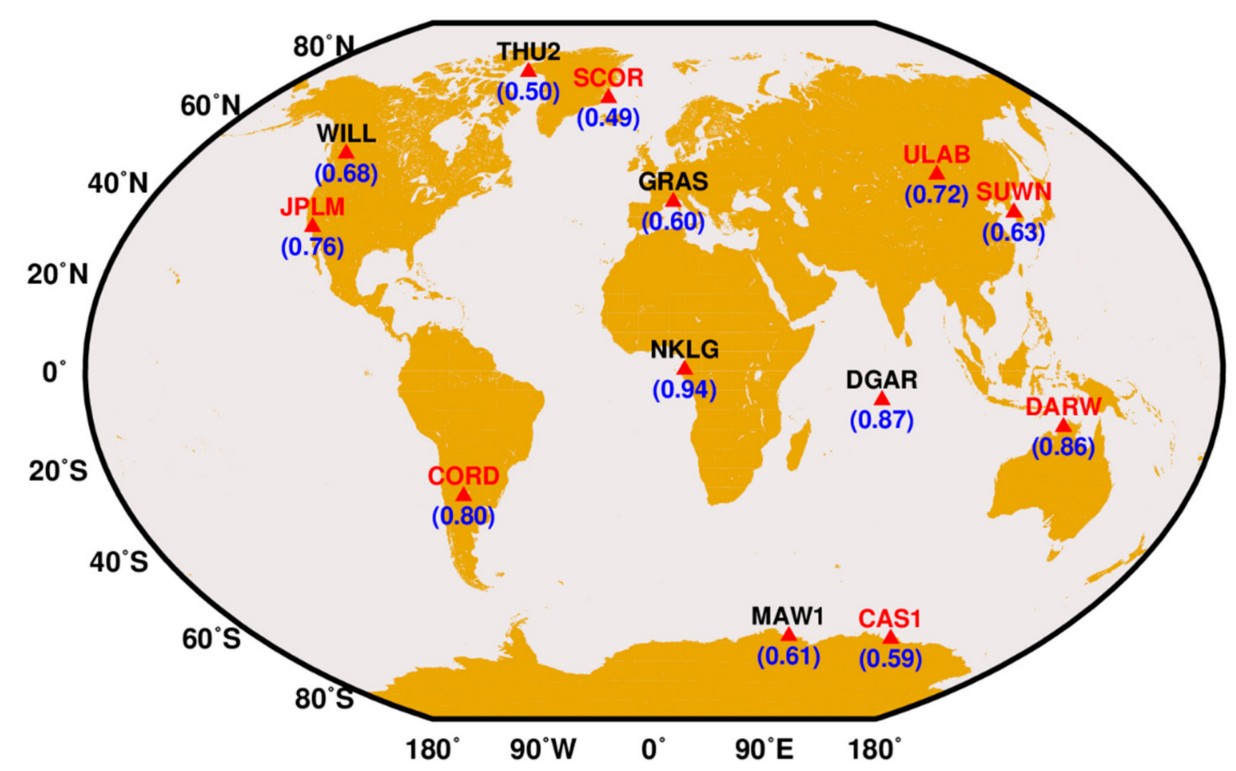

Figure 5. Geographic locations of the 13 GNSS stations, which are marked by red triangles. The seven additional GNSS stations (JPLM: 34.2 N, SCOR: 70.4 N, ULAB: 47.8 N, SUWN: 37.2 N, DARW: 12.8 S, CORD: $31.5 \mathrm{~S}$, and CAS1: $66.2 \mathrm{~S}$ ) considered for correlation analysis with different latitude regions are indicated in red font. The numbers in parentheses are the $R$ value between and rDCB RMS for three years.

Table 2. The receiver and antenna types at the 7 additional GNSS stations from 2014 to 2016.

\begin{tabular}{ccc}
\hline Site Name & Receiver Type & Antenna Type \\
\hline JPLM & JPS EGGDT & AOAD/M_T NONE \\
SCOR & ASHTECH UZ-12 & ASH701941.B SCIS \\
ULAB & JAVAD TRE_G3TH DELTA & JAV_RINGANT_G3T NONE \\
SUWN & TRIMBLE NETR9 & TRM59800.80 SCIS \\
DARW & LEICA GRX1200GGPRO & ASH700936D_M NONE \\
CORD & JAVAD TRE_G3TH DELTA & TPSCR.G3 NONE \\
CAS1 & TRIMBLE NETR9 & LEICA25.R3 LEIT \\
\hline
\end{tabular}

\section{Discussion}

There was a high correlation between TEC and rDCB stability at low latitudes. For a better analysis of these characteristics, we considered two GNSS stations (BOGT and KOUR) located in South America, as shown in Figure 6. The geographic latitudes of BOGT and KOUR stations at the equatorial region are about 4.64 and 5.25 degrees, respectively. For three years from 2014 to 2016, the BOGT and KOUR sites had operated GNSS receivers (JAVAD TRE_3 DELTA and SEPT POLARX4) of different type models. The rDCB may be different depending on the receiver type. Even the same receiver models have different $\mathrm{rDCB}$ values $[18,30]$. Therefore, it may be important to investigate the correlation in the GNSS stations with different receiver models.

Figure $6 \mathrm{~b}$ shows the time series of the VTEC and rDCB RMS*50 at BOGT (top panel) and KOUR (lower panel), respectively. The blue solid line denotes the changes in VTEC for three years from 2014 to 2016. Purple circles represent the changes in the rDCB RMS*50. The linear correlation between those variables is displayed in the upper right corner of each panel.

The VTEC values have been decreasing gradually since 2014, as seen in the upper panel of Figure $6 \mathrm{~b}$. The maximum value of the TEC peaked in 2014, and reached its minimum value at the end of 2016. We can also observe a clear annual and semi-annual variation of the VTEC. The VTEC was higher at the equinoxes than at the solstices during the period from 2014 to 2016. The magnitude of 
TEC was highest at the March equinox during this period, and lowest at the June solstice. This is fully consistent with the results reported by Akala et al. [32]. They attribute to increasing in ion production rate in the equinox and low ionization resulting from reduced production rate in the solstice during this period. As shown in the upper panel of Figure $6 \mathrm{~b}$, the changes in rDCB RMS were very similar to the changes in VTEC. We calculated that the value of $R$ between VTEC and rDCB RMS was about 0.90 .

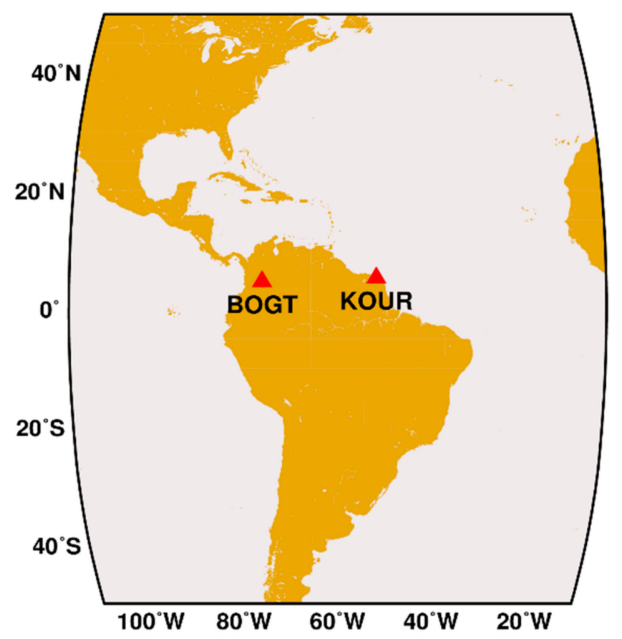

(a)

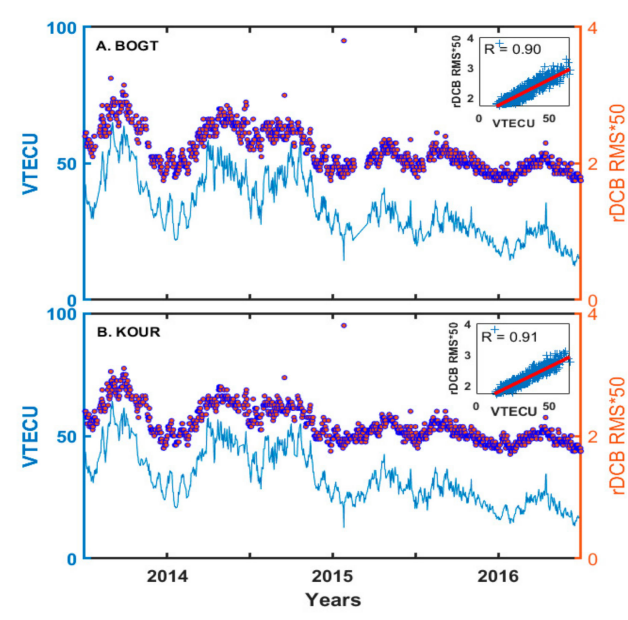

(b)

Figure 6. (a) Locations of the two GNSS stations (BOGT and KOUR) in South America, which are marked by red triangles. (b) Time series of VTEC and rDCB RMS for three years: (top) BOGT, (bottom) KOUR. The blue solid lines indicate VTEC changes. Purple circles denote the rDCB RMS. The small panels plot a linear correlation between VTEC and rDCB RMS. The ' $R$ ' character in the small panels indicates the Pearson's correlation coefficient.

From the results in the bottom panel of Figure 5b, the KOUR station with $R$ of 0.91 shows very similar features to the BOGT station. Changes in VTEC could be similar because the two stations have similar latitudes and are located close to each other. It is noted that although the two stations use different GNSS receiver models, the changes in rDCB RMS are similar. This suggests that the ionospheric TEC at low latitudes and equatorial regions is strongly related to the stability of the rDCB.

In addition, we have analyzed the changes in rDCB and rDCB RMS as the model of the GNSS receiver is changed. Figure 7 shows the time series of $\mathrm{rDCB}$ and $\mathrm{rDCB}$ RMS at the ALIC GNSS station (GLAT: $23.7^{\circ}$ S, GLON: $133.8^{\circ} \mathrm{E}$ ) from 2014 to 2016 . The red solid lines represent the polynomial-fitted curve of the data. As shown in the upper panel of Figure 7, the rDCB values have been stable since 2014 with only small changes. However, they show a large discrepancy in early 2016. This is due to the replacement of the GNSS receiver. In Figure 7, the blue vertical dotted line indicates when the receiver was replaced. The large change in the $\mathrm{PDCB}$ is exactly in line with receiver replacement. The GNSS receiver in the ALIC station was changed from the 'LEICA GRX1200GGPRO' to the 'LEICA GR25' model in February 2016. The rDCB value of the ALIC station changed from about 12 to $21 \mathrm{~ns}$ with the receiver replacement. The lower panel of Figure 7 shows the rDCB RMS time series for three years. The rDCB RMS reaches its maximum value in early 2014, and then tends to decrease gradually. In addition, rDCB RMS has periodic and time-varying features. It is noteworthy that the rDCB RMS of the ALGO station did not change significantly despite the replacement of the receiver in early 2016. Changes in the rDCB RMS, even after receiver replacement, have continuity. This indicates that the stability of the rDCB can also be independent of the GNSS receiver model. 


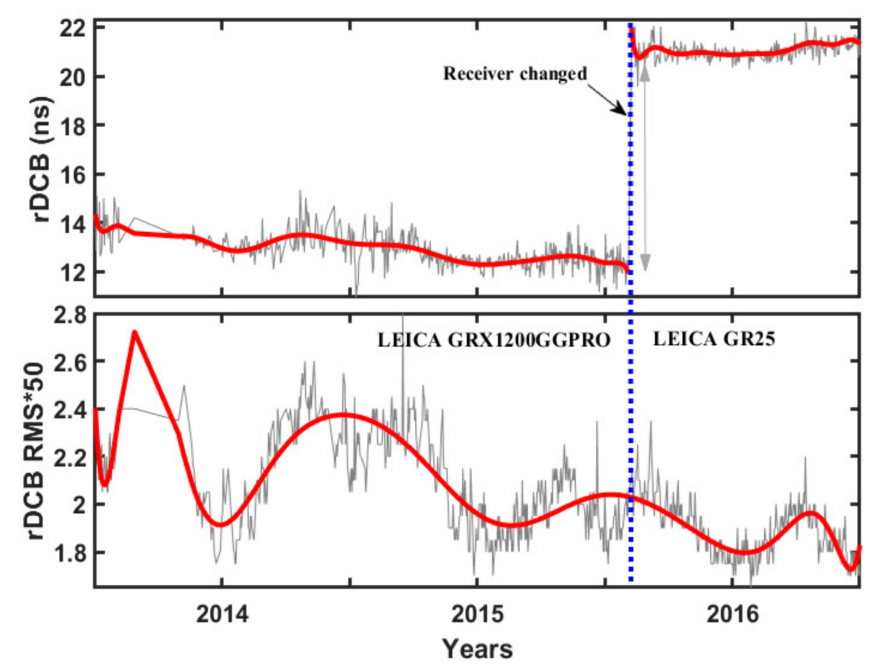

Figure 7. Time series of $\mathrm{rDCB}$ and rDCB RMS at the ALIC GNSS station. The grey solid lines are the raw data, and the red solid lines represent the polynomial curve fitted to the raw data. The blue vertical dotted line indicates the point in time when the GNSS receiver was replaced.

To investigate the correlation between VTEC changes and rDCB stability before and after replacing the receiver, we plotted the time series of VTEC and rDCB RMS*50 for the same period of time. In Figure 8, the blue solid line and the brown solid line represent the VTEC and rDCB RMS changes, respectively. They represent a polynomial fitting of the data. The time series of the two variables show similar features, such as periodicity and damping. $R$ between the two variables is about 0.92 , which means that there is a very strong correlation. Therefore, we found that the ionospheric TEC and rDCB stability have a very strong correlation even after the replacement of the receiver.

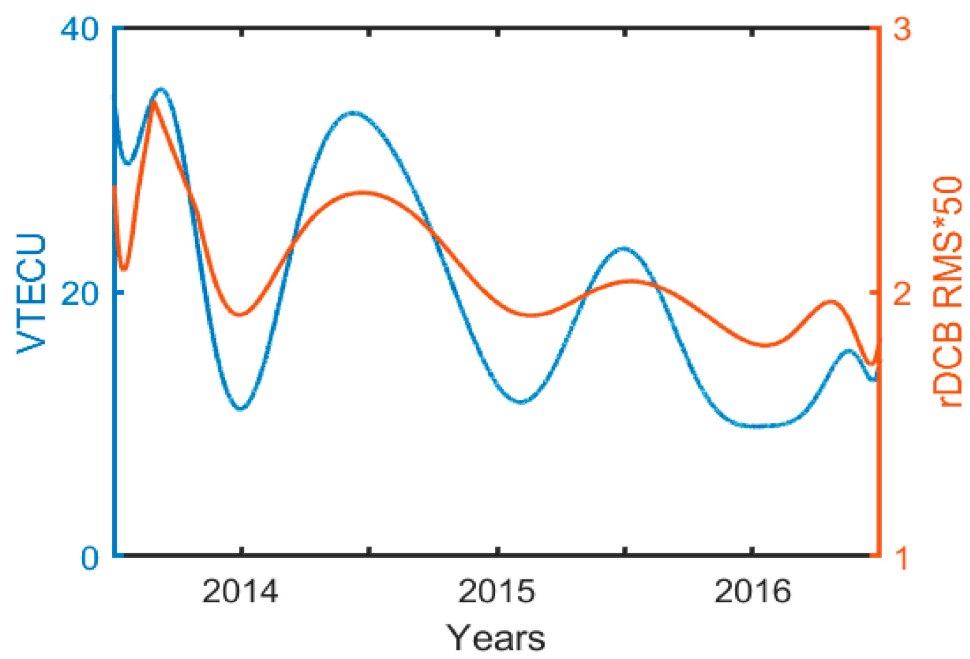

Figure 8. Time series of VTEC and rDCB RMS with a polynomial fit of 10 degrees for the ALIC GNSS station. The blue solid line is VTEC, and the brown solid line represents rDCB RMS.

According to Kao et al. [33], there are various factors affecting GNSS rDCB estimation accuracy. They demonstrated that the estimation accuracy of rDCB is lower in the low-latitude regions because TEC changes in low-latitude regions are more complicated than those in mid-latitude regions. In this study, we showed that the values of $R$ between TEC and rDCB RMS were also larger in low-latitude regions than those in mid-latitude or high-latitude regions. Besides, this may be explained that rDCB stability is poor due to the complex structure of ionospheric TEC in low-latitude regions. It can affect the accuracy of ionospheric TEC estimation. Eventually, the estimation of real-rDCB can increase 
the accuracy of TEC estimation, which will contribute to improving the position accuracy of the GNSS users.

\section{Conclusions}

In this study, we have investigated the relationship between VTEC and $\mathrm{rDCB}$, and VTEC and rDCB stability, during the three years from 2014 to 2016. Pearson's linear correlation values were considered in order to quantify the relationships between the variables. In addition, because the magnitude of the GNSS TEC has a latitudinal dependency, we used data from 13 GNSS stations located in low-, middle-, and high-latitude regions.

The time series of VTEC, rDCB, and rDCB RMS at each GNSS station from 2014 to 2016 were analyzed. We could see clear periodic features in time series at different latitudes. The most prominent features were the annual and semiannual variations. The semiannual variations were more significant in low-latitude and mid-latitude regions than in high-latitude regions. In addition, these characteristics of $\mathrm{rDCB}$ and $\mathrm{rDCB}$ RMS have a similarity with those of VTEC, at all stations. By calculating $R$ between the variables, it can be shown that the correlation between TEC and rDCB is the smallest in low-latitude regions. The mid-latitude regions exhibit the maximum value of $R$. This can be related to ionospheric TEC irregularities with different latitude regions. In contrast, $R$ values between TEC and rDCB RMS were greater in low-latitude regions, which can be associated with the magnitude of TEC. A strong positive correlation $(\geq 0.90)$ between TEC and rDCB RMS was also observed at the two additional GNSS stations in low-latitude regions. This suggests that the correlation between the two different variables has a clear latitudinal dependence.

The rDCB values showed large differences before and after replacing GNSS receivers, which is consistent with earlier studies. The rDCB RMS has periodic and time-varying features, and is not changed by the replacement of the receiver. Changes in the rDCB RMS have continuity even after receiver replacement. $R$ between TEC and rDCB RMS after replacing the receiver was 0.92 , indicating a very strong correlation. Therefore, it can be noted that $\mathrm{rDCB}$ RMS is not related to the GNSS receiver model. As a result, we found that ionospheric TEC and $\mathrm{HDCB}$ stability still have a very strong correlation. Estimation of stable rDCB can contribute to improving the accuracy of the GNSS ionospheric TEC. Therefore, applying the correlation between ionospheric TEC changes and rDCB stability in different latitude regions to the ionospheric TEC will allow for more accurate monitoring of GNSS ionospheric TEC.

Author Contributions: Methodology, B.-K.C.; software, B.-K.C.; validation, B.-K.C. and D.-H.S.; investigation, B.-K.C.; writing-original draft preparation, B.-K.C.; writing—review and editing, B.-K.C., D.-H.S. and S.J.L.; visualization, B.-K.C. and D.-H.S.

Funding: This research was funded by the Primary Project of the Korea Astronomy and Space Science Institute (2019185003).

Acknowledgments: The authors would like to thank the IGS for providing GNSS data and IONEX GIM products. The authors would also like to take the opportunity to thank the anonymous reviewers for their constructive comments.

Conflicts of Interest: The authors declare no conflict of interest.

\section{References}

1. Coco, D.; Coker, C.; Dahlke, S.; Clynch, J. Variability of GPS satellite differential group delay biases. IEEE Trans. Aerosp. Electr. Syst. 1991, 27, 931-938. [CrossRef]

2. Sardon, E.; Rius, A.; Zarraoa, N. Estimation of the transmitter and receiver differential biases and the ionospheric total electron content from Global Positioning System observations. Radio Sci. 1994, 29, 577-586. [CrossRef]

3. Otsuka, Y.; Ogawa, T.; Saito, A.; Tsugawa, T.; Fukao, S.; Miyazaki, S. A new technique for mapping of total electron content using GPS network in Japan. Earth Planets Space 2002, 54, 63-70. [CrossRef]

4. Ma, X.; Maruyama, T.; Ma, G.; Maruyama, T. Determination of GPS receiver differential biases by neural network parameter estimation. Radio Sci. 2005, 40, RS1002. [CrossRef] 
5. Keshin, M. A new algorithm for single receiver DCB estimation using IGS TEC maps. GPS Solut. 2012, 16, $283-292$. [CrossRef]

6. Montenbruck, O.; Hauschild, A.; Steigenberger, P. Differential Code Bias estimation using multi-GNSS observations and global ionosphere maps. Navigation 2014, 61, 191-201. [CrossRef]

7. Li, Z.; Yuan, Y.; Wang, N.; Hernandez-Pajares, M.; Huo, X. SHPTS: Towards a new method for generating precise global ionospheric TEC map based on spherical harmonic and generalized trigonometric series functions. J. Geod. 2015, 89, 331-345. [CrossRef]

8. Zhang, B.; Teunnissen, P.J.G.; Yuan, Y. On the short-term temporal variations of GNSS receiver differential phase biases. J. Geod. 2017, 91, 563-572. [CrossRef]

9. Liu, T.; Zhang, B.; Yuan, Y.; Li, Z.; Wang, N. Multi-GNSS triple-frequency differential code bias (DCB) determination with precise point positioning (PPP). J. Geod. 2019, 93, 765-784. [CrossRef]

10. Wilson, B.; Mannucci, A. Extracting ionospheric measurements from GPS in the presence of Anti-Spoofing. In Proceedings of the ION GPS-94, Institute of Navigation, Salt Lake City, UT, USA, 20-23 September 1994; pp. 1599-1608.

11. Hernandez-Pajares, M.; Juan, J.M.; Sanz, J.; Orus, R.; Garcia-Rigo, A.; Feltens, J.; Komjathy, A.; Schaer, S.C.; Krankowski, A. The IGS VTEC maps: A reliable source of ionospheric information since 1998. J. Geod. 2009, 83, 263-275. [CrossRef]

12. Warnant, R. Reliability of the TEC computed using GPS measurements-The problem of hardware biases. Acta Geod. Geophys. Hung. 1997, 32, 451-459.

13. Coster, A.; Williams, J.; Weatherwax, A.; Rideout, W.; Herne, D. Accuracy of GPS total electron content: GPS receiver bias temperature dependence. Radio Sci. 2013, 48, 190-196. [CrossRef]

14. Yasyukevich, Y.V.; Mylnikova, A.A.; Kunitsyn, V.E.; Padokhin, A.M. Influence of GPS/GLONASS differential code biases on the determination accuracy of the absolute total electron content in the ionosphere. Geomagn. Aeron. 2015, 55, 763-769. [CrossRef]

15. Zhang, B.; Teunnissen, P.J.G. Characterization of multi-GNSS between-receiver differential code biases using zero and short baselines. Sci. Bull. 2015, 60, 1840-1849. [CrossRef]

16. Mannucci, A.J.; Iijima, B.A.; Sparks, L.; Pi, X.; Wilson, B.D.; Lindqwister, U.J. Assessment of global TEC mapping using a three-dimensional electron density model. J. Atmos. Sol. Terr. Phys. 1999, 61, 1227-1236. [CrossRef]

17. Zhang, W.; Zhang, D.H.; Xiao, Z. The influence of geomagnetic storms on the estimation of GPS instrumental biases. Ann. Geophys. 2009, 27, 1613-1623. [CrossRef]

18. Choi, B.K.; Lee, S.J. The influence of grounding on GPS receiver differential code biases. ASR 2018, 62, 457-463. [CrossRef]

19. Mannucci, A.J.; Wilson, B.D.; Yuan, D.N.; Ho, C.H.; Lindqwister, U.J.; Runge, T.F. A global mapping technique for GPS-derived ionospheric total electron content measurements. Radio Sci. 1998, 33, 565-582. [CrossRef]

20. Dow, J.M.; Neilan, R.E.; Rizos, C. The International GNSS Service in a changing landscape of Global Navigation Satellite Systems. J. Geod. 2009, 83, 191-198. [CrossRef]

21. Zhang, D.; Shi, H.; Jin, Y.; Zhang, W.; Hao, Y.; Xiao, Z. The variation of the estimated GPS instrumental bias and its possible connection with ionospheric variability. Sci. China Tech. Sci. 2014, 57, 67-79. [CrossRef]

22. Themens, D.R.; Jayachandran, P.T.; Langley, R.B. The nature of GPS differential receiver bias variability: An examination in the polar cap region. J. Geophys. Res. Space Phys. 2015, 120, 8155-8175. [CrossRef]

23. Mazzella, A.J. Plasmasphere effects for GPS TEC measurements in North America. Radio Sci. 2009, 44, RS5014. [CrossRef]

24. Conte, J.F.; Azpilicueta, F.; Brunini, C. Accuracy assessment of the GPS-TEC calibration constants by means of a simulation technique. J. Geod. 2011, 85, 707-714. [CrossRef]

25. Schaer, S.; Beutler, G.; Mervart, L.; Rothacher, M.; Wild, U. Global and regional ionosphere models using the GPS double difference phase observable. In Proceedings of the IGS Workshop 1995, GFZ, Potsdam, Germany, 15-18 May 1995; pp. 77-92.

26. Wielgosz, P.; Grejner-Brzezinska, D.; Kashani, I. Regional ionosphere mapping with kriging and multiquadratic methods. J. GPS 2003, 2, 48-55. [CrossRef]

27. Zhao, B.; Wan, W.; Liu, L.; Mao, T.; Ren, Z.; Wang, M.; Christensen, A.B. Features of annual and semiannual variations derived from the global ionospheric maps of total electron content. Ann. Geophys. 2008, 25, 2513-2527. [CrossRef] 
28. Schober, P.; Boer, C.; Schwarte, L.A. Correlation Coefficients: Appropriate Use and Interpretation. Anesth. Analg. 2018, 126, 1763-1767. [CrossRef] [PubMed]

29. Perkins, F.W. Ionospheric irregularities. Rev. Geophys. 1975, 13, 884. [CrossRef]

30. Fejer, B.G.; Kelley, M.C. Ionospheric irregularities. Rev. Geophys. Space Phys. 1980, 18, 401-454. [CrossRef]

31. Guo, J.; Li, W.; Liu, X.; Kong, Q.; Zhao, C.; Guo, B. Temporal-Spatial Variation of Global GPS Derived Total Electron Content, 1999-2013. PLoS ONE 2015, 10, e0133378. [CrossRef]

32. Akala, A.O.; Seemala, G.K.; Doherty, P.H.; Valladares, C.E.; Carrano, C.S.; Espinoza, J.; Oluyo, S. Comparison of equatorial GPS-TEC observations over an African station and American station during the minimum and ascending phases of solar cycle 24. Ann. Geophys. 2013, 31, 2085-2096. [CrossRef]

33. Kao, S.; Chen, W.; Weng, D.J.; Ji, S.Y. Factors affecting the estimation of GPS receiver instrumental biases. Surv. Rev. 2013, 45, 59-67. [CrossRef]

(C) 2019 by the authors. Licensee MDPI, Basel, Switzerland. This article is an open access article distributed under the terms and conditions of the Creative Commons Attribution (CC BY) license (http://creativecommons.org/licenses/by/4.0/). 